\title{
Erythrocyte Deformability in Children With Autism Spectrum Disorder: Correlation With Clinical Features
}

\author{
T. JASENOVEC ${ }^{1}$, D. RADOSINSKA ${ }^{2}$, H. CELUSAKOVA ${ }^{1}$, D. FILCIKOVA ${ }^{1}$, \\ K. BABINSKA ${ }^{1}$, D. OSTATNIKOVA ${ }^{1}$, J. RADOSINSKA ${ }^{1,3}$
}

${ }^{1}$ Institute of Physiology, Faculty of Medicine, Comenius University in Bratislava, Bratislava, Slovak Republic, ${ }^{2}$ Faculty of Natural Sciences, Comenius University in Bratislava, Bratislava, Slovak Republic, ${ }^{3}$ Centre of Experimental Medicine, Institute for Heart Research, Slovak Academy of Sciences, Bratislava, Slovak Republic

Received March 21, 2019

Accepted September 26, 2019

\section{Summary}

Biomechanical properties of erythrocytes play an important role in health and disease. Deformability represents intrinsic property of erythrocytes to undergo deformation that is crucial for their passage through the narrow capillaries. The erythrocyte damage can lead to compromised tissue perfusion and consequently play a role in the pathogenesis of various diseases including neurological ones. Data available in databases indicate that erythrocytes in autism spectrum disorder (ASD) are altered. This may affect the clinical symptoms of ASD. The aim of our study was to determine erythrocyte deformability in 54 children with ASD and correlate it with clinical symptoms. We found significant negative correlation between erythrocyte deformability and score in $\mathrm{C}$ domain of the Autism Diagnostic Interview-Revised (ADI-R) diagnostic tool describing the measure of restrictive, repetitive, and stereotyped behaviors and interests, mainly observable in $\mathrm{C} 1$ and $\mathrm{C} 2$, but not in C3 and C4 subdomains. This supports the findings of other authors and suggest that behavioral domain $\mathrm{C}$ comprises of more subcategories with different underlying etiology. Our results also indicate that abnormalities in erythrocyte deformability may be involved in ASD pathomechanisms and contribute to its clinical manifestation. Further research is necessary to bring more data and identify erythrocyte deformability as prognostic biomarker in ASD.

\section{Key words}

Erythrocytes - Erythrocyte deformability - Autism spectrum disorder • Autism Diagnostic Interview-Revised

\section{Corresponding author}

J. Radosinska, Institute of Physiology, Faculty of Medicine, Comenius University in Bratislava, Sasinkova 2, 81372 Bratislava, Slovak Republic. E-mail: jana.radosinska@fmed.uniba.sk

\section{Introduction}

Autism spectrum disorder (ASD) is a group of lifelong neurodevelopmental disorders that manifests mostly in the first three years of life. ASD are defined by their core symptoms including difficulties in social communication and social interaction; and restricted, repetitive patterns of behavior, interests or activities. Aside from typical behavioral characteristics, individuals with ASD may also suffer from a wide range of somatic disturbances, e.g. immunological (Edmiston et al. 2017), gastrointestinal (Kushak et al. 2016), sleep disorders (Richdale and Schreck 2009) and others (Muskens et al. 2017), with much higher rates than general population. Associated medical conditions negatively affect the core symptoms of ASD, and increase the probability of challenging behaviors. It is not known yet, whether the health disturbances are a cause, consequence, comorbidity or coincidence of ASD (Mazurek 2016). At present time, ASD represent a serious health problem due to their increasing prevalence, serious clinical presentation, unclear etiology and absence of causal treatment (Lyall et al. 2017).

Red blood cells (RBCs) are the most numerous blood elements. Their main function is transport of 
oxygen to all tissues in human body. To accomplish this function, RBCs with the diameter of $7.2 \mu \mathrm{m}$ have to pass through capillaries, which have smaller diameters (3-7 $\mu \mathrm{m}$ ) than RBCs themselves (Lim et al. 2018). To do so, RBCs must be able to repeatedly change their shape without taking any damage. This ability is referred to as deformability of RBCs. There is much evidence for a long time, that RBC deformability is crucial for effective microcirculation and adequate delivery of oxygen to the tissues (Schmid-Schonbein 1976). Moreover, due to their short lifespan, RBCs may serve as indicators of health, and could play a role in tracking disease progression and treatment (Diez-Silva et al. 2010, Pretorius et al. 2016). Worsening of RBC deformability plays an important role in the ethiopathogenesis of various diseases including cardiovascular (Radosinska and Vrbjar 2016) and neurological ones (Chang et al. 2007, Tanashyan et al. 2018).

Various research teams observed the presence of echinocytes, anisocytes, poikilocytes, dacrocytes, schistocytes and other abnormal shapes of the RBCs in peripheral blood of autistic children (Nabila et al. 2012, Ciccoli et al. 2013, Bolotta et al. 2018). Furthermore, abnormal shape of RBCs was identified as one of three unrecognized, but typical RBC-related biological markers in autism (together with $\mathrm{RBC}$ membrane oxidative damage, and $\beta$-actin alterations) (Ciccoli et al. 2013). The RBC shape morphology changes are related to alterations of RBC deformability (Diez-Silva et al. 2010). Indeed, lower deformability of RBCs was found in children with ASD when compared with neurotypical children (László et al. 2013). Nevertheless, to the best of our knowledge, no study has described relationship between RBC deformability and severity of clinical manifestation in ASD yet. We presumed that severity of ASD symptoms may be also influenced by quality of RBCs, and specifically by their deformability. Thus, the aim of our study was to determine RBC deformability in a sample of Slovak children suffering from ASD and to assess relationship between RBC deformability and severity of clinical symptoms in autistic children.

\section{Methods}

\section{Study design}

Our study involved 54 non-verbal children diagnosed with ASD (44 boys and 10 girls). The median age of children was 3.5 years (minimum 2.6, maximum 6.9 years). Children with suspect ASD were diagnosed at
Academic Research Centre for Autism, Institute of Physiology, Faculty of Medicine, Comenius University in Bratislava using Autism Diagnostic Observation Schedule - second edition (ADOS-2) and Autism Diagnostic Interview-Revised (ADI-R) to confirm the diagnosis. All participants were administered module 1 of ADOS-2. This module was designed for children, who are over 31 months old and do not consistently use phrase speech. Right after diagnostic procedures, venous blood was drawn from each participant.

The study was approved by the Ethics Committee of Faculty of Medicine, Comenius University in Bratislava, Slovak Republic. Informed consent was obtained from all subjects or their caregivers included in the study. The study conformed to the code of ethics stated in the Declaration of Helsinki.

ASD symptoms assessment

ASD was diagnosed according to DSM-V criteria (American Psychiatric Association 2013) as described previously (Babinská et al. 2017). We used ADI-R (Lord et al. 1994) and ADOS-2 (Lord et al. 2012) as diagnostic tools. ADI-R is a structured interview conducted with a parent. Each symptom of ASD can be quantified on scale 0-3 (0-behavior not present, 3 -extreme severity of behavior). ADI-R consists of five main sections. Three of them focus on the core deficits of ASD. The diagnostic algorithm used in our study consists of three main domains. Domain A evaluates qualitative abnormalities in reciprocal social interaction. There are 4 subdomains (A1-4) which score failure to use nonverbal behaviors to regulate social interaction (A1), failure to develop peer relationships (A2), lack of shared enjoyment (A3) and lack of socioemotional reciprocity (A4). Domain B evaluates qualitative abnormalities in communication and has 4 subdomains (B1-4). B1 is used to rate lack of, or delay in, spoken language and failure to compensate through gesture and B4 for lack of varied spontaneous make-believe or social imitative play. B2 relative failure to initiate or sustain conversational interchange and B3 (stereotyped, repetitive or idiosyncratic speech) can only be evaluated in verbal individuals. Domain $\mathrm{C}$ is also composed of 4 subdomains (C1-4) and is used to evaluate restricted, repetitive, and stereotyped patterns of behavior. $\mathrm{C} 1$ scores encompassing preoccupation or circumscribed pattern of interest, $\mathrm{C} 2$ apparently compulsive adherence to nonfunctional routines or rituals, $\mathrm{C} 3$ stereotyped and repetitive motor mannerisms and $\mathrm{C} 4$ preoccupations with part of objects or non- 
functional elements of material. ADOS-2 is a diagnostic method of direct observation of the children. This scale evaluates core ASD symptoms in two domains: social affect, and restricted and repetitive behavior scores. Child is assigned to one of 5 modules (Toddler, M1-4) depending on expressive language level and age of individual with suspected ASD.

\section{Deformability of red blood cells}

Venous blood was taken into EDTA-containing vacutainer tubes right after diagnostic tests. There are many ways to determine RBC deformability, the filtration is one of the oldest methods (Kim et al. 2015). It considers behavior of multiple RBCs, not only single cells. RBC deformability was determined by filtration method as described previously (Radosinska et al. 2016). Briefly, blood was centrifuged at $1150 \mathrm{~g}$ for $5 \mathrm{~min}$ at $4{ }^{\circ} \mathrm{C}$ immediately after venous blood sampling. The plasma, buffy coat and upper $20 \%$ of RBCs were removed and discarded. Rest of the RBCs was washed in saline solution for three times. Washed RBCs were diluted in Cellpack solution (diluent for Sysmex blood analyzer, $1: 1000, \mathrm{~V}: \mathrm{V})$ and filtered by centrifugation at $175 \mathrm{~g}$ through membrane filters with $5 \mu \mathrm{m}$ pores in diameter (Ultrafree-MC SV Centrifugal Filter; Merck Millipore Ltd.). RBC deformability was calculated as a ratio of filtered RBCs to their number before filtration. Together with RBC counting, Sysmex blood analyzer also provided information about mean cell volume (MCV) that reflects the size of individual RBC.

\section{Statistical analysis}

Data are presented as means \pm standard deviations (SD) when normally distributed, or as medians with minimum and maximum values when distribution of data was not Gaussian. Normality of data was analyzed by D'Agostino and Pearson normality test. Spearman's correlation test (or Pearson's correlation test if data passed the normality test) was used to correlate RBC deformability, $\mathrm{MCV}$, and behavioral parameters of ADI-R and ADOS-2. Values $\mathrm{p}<0.05$ were considered as statistically significant. As our study was explorative and we did not test specific hypothesis, we did not perform multiple testing correction and our $p$ values are unadjusted. GraphPad Prism 7 software was used for all statistical analyses.

\section{Results}

An average RBC deformability was
$72.81 \pm 5.60 \%$ in ASD children that participated in our study. We observed neither significant difference between boys and girls $(72.85 \pm 5.89 \%$ in boys, $72.60 \pm 4.35$ in girls, p=0.9, Fig. 1) in RBC deformability, nor correlation between $\mathrm{RBC}$ deformability and the age of participants $(r=0.15, p=0.27$, Fig. 2$)$.

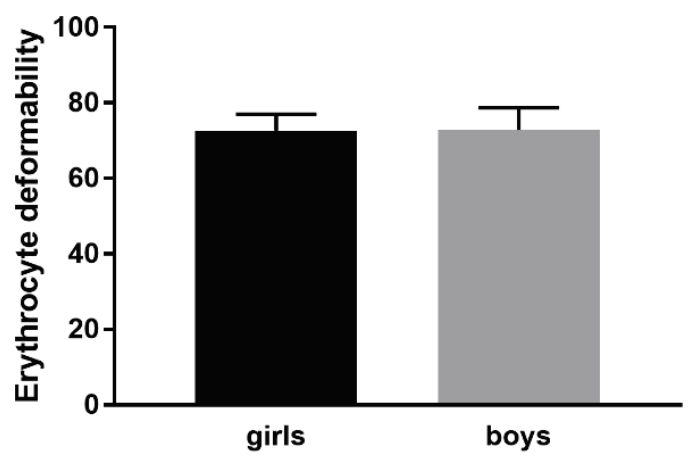

Fig. 1. Erythrocyte deformability in boys and girls in our group of participants suffering from autism spectrum disorder. The data are presented as means \pm SD. Sample size: boys $-n=44$, girls $-n=10$.

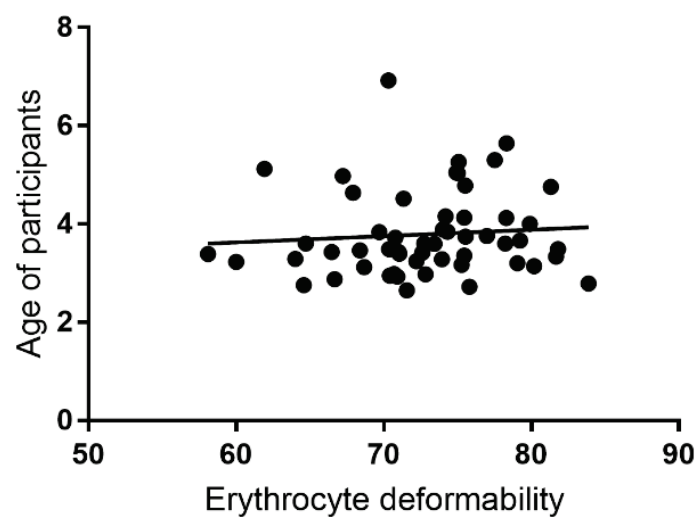

Fig. 2. Correlation between erythrocyte deformability and age of participants suffering from autism spectrum disorder $(r=0.15$, $p=0.27$ ).

We found a significant negative correlation between RBC deformability and $\mathrm{C}$ domain of ADI-R diagnostic tool. Children with lower deformability of RBCs suffered from more severe symptoms in the area of restrictive, repetitive, and stereotyped behaviors and interests (Fig. 3). Similar significant negative correlations were found in two $\mathrm{C}$ subdomains ( $\mathrm{C} 1$ and $\mathrm{C} 2$ ). The less deformable RBCs in ASD children were associated with more significant clinical manifestation in form of encompassing preoccupation or circumscribed pattern of interest $(\mathrm{C} 1)$ and an apparently compulsive adherence to non-functional routines or rituals (C2). No relationship between RBC deformability and A (Qualitative 
Abnormalities in Reciprocal Social Interaction), B (Qualitative Abnormalities in Communication) domains, and $\mathrm{C} 3$ (Stereotyped and repetitive motor mannerisms) and C4 (Preoccupations with part of objects or non-functional elements of material) subdomains was detected. Summary of correlations between the individual domains and subdomains of ADI-R diagnostic tool and RBC deformabilities in children with ASD is presented in Table 1. Figure 4 illustrates scores of participants in individual main domains and $\mathrm{C}$ subdomains of ADI-R diagnostic tool.

An average $\mathrm{MCV}$ was $75.31 \pm 4.52 \mathrm{fl}$ in our participants. We observed no statistically significant correlation between $\mathrm{MCV}$ and $\mathrm{RBC}$ deformability $(\mathrm{r}=-0.20, \mathrm{p}=0.15)$. Similarly, we found no relation between MCV and scores in $\mathrm{C}$ domain $(\mathrm{r}=-0.08, \mathrm{p}=0.58)$ as well as in $\mathrm{C} 1(\mathrm{r}=-0.11, \mathrm{p}=0.44)$ and $\mathrm{C} 2(\mathrm{r}=0.01$, $\mathrm{p}=0.97$ ) subdomains of ADI-R diagnostic tool.

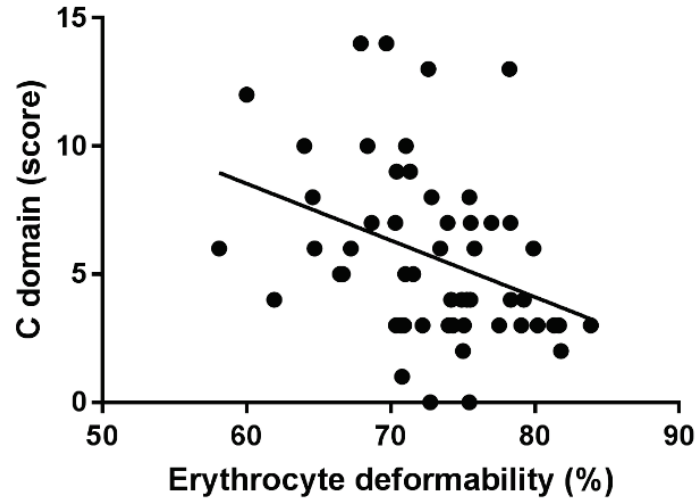

Fig. 3. Correlation between erythrocyte deformability and score in $\mathrm{C}$ domain of ADI-R diagnostic tool. $\mathrm{C}$ domain consists of four subdomains, each consisting of two items included in the diagnostic algorithm. Each item represents distinct symptom for ASD and is usually rated on the scale $0-3$. Score in C domain was calculated according to the ADI-R manual (using either summation or maximum value), maximal score can theoretically reach 18. Statistical significance: Spearman $r=-0.4$ (95\% confidence interval -0.608 to -0.1397$), p=0.0027$. Abbreviation: ADI-R - Autism Diagnostic Interview-Revised.

Table 1. Correlations between the individual domains and subdomains of ADI-R diagnostic tool and erythrocyte deformabilities in autistic children.

\begin{tabular}{lccc}
\hline Domains of ADI-R & $\mathbf{r}$ & p value & Significance \\
\hline A domain & -0.21 & 0.1241 & $\mathrm{~ns}$ \\
B domain & -0.09 & 0.5259 & $\mathrm{~ns}$ \\
C domain & $\mathbf{- 0 . 4 0}$ & $\mathbf{0 . 0 0 2 7}$ & $* *$ \\
C1 subdomain & $\mathbf{- 0 . 4 3}$ & $\mathbf{0 . 0 0 1 4}$ & $*$ \\
C2 subdomain & $\mathbf{- 0 . 3 0}$ & $\mathbf{0 . 0 2 9 0}$ & $\mathrm{ns}$ \\
C3 subdomain & 0.05 & 0.7463 & $\mathrm{~ns}$ \\
C4 subdomain & -0.21 & 0.1244 & \\
\hline
\end{tabular}

Abbreviation: ADI-R - Autism Diagnostic Interview-Revised, $r$ - Spearman's correlation coefficient, ${ }^{*} \mathrm{p}<0.05,{ }^{* *} \mathrm{p}<0.01$.

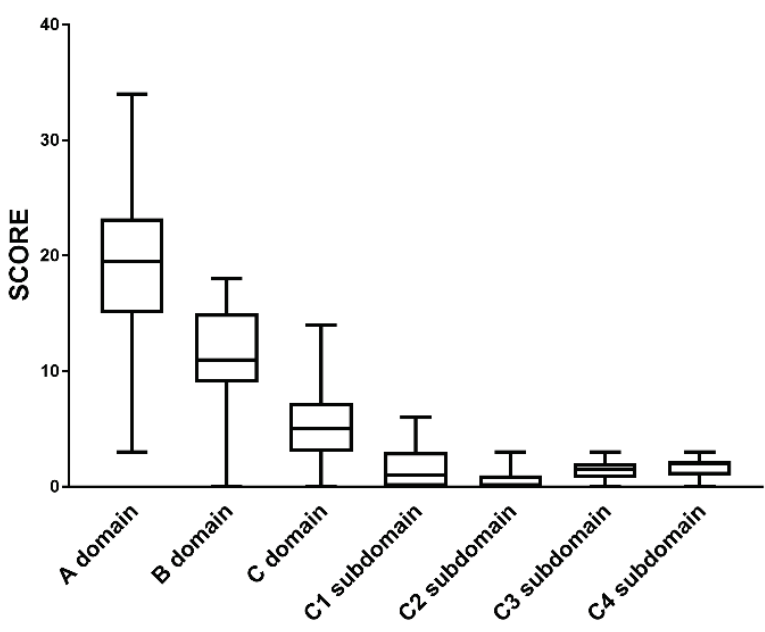

Fig. 4. Scores of participants in individual main domains and C subdomains (data in A and B domain and C4 subdomain passed the normality test).

\section{Discussion}

In our study, we found a link between the ability of RBCs to change their shape, i.e. RBC deformability and severity of several behavioral parameters in children with ASD. In capillaries, where RBCs must deform to enter and transit vessels that are smaller than the resting $\mathrm{RBC}$ diameter, their deformability is the most important factor affecting the blood flow (Parthasarathi and Lipowsky 1999, Baskurt and Meiselman 2003). Impaired RBC deformability may lead to lowered tissue oxygen supply in various organs, central nervous system included, which may have an adverse effect on behavior of children with ASD. Nevertheless, it remains unclear to what extent the impaired RBC deformability in ASD is 
directly responsible for more severe clinical manifestation of ASD, or the decreased RBC deformability and more severe ASD phenotype have the same causal origin.

One mechanism, that may explain changes of $\mathrm{RBC}$ deformability in autistic children, is an effect of free radicals on the RBCs. Imbalance in oxidative status is a common factor impairing deformability of the RBCs in numerous pathological conditions such as diabetes mellitus, thromboembolic stroke, rheumatoid arthritis, Alzheimer and Parkinson's disease or systemic inflammation (Pretorius et al. 2016). Systemic proinflammatory status (Babinská et al. 2014) and increased oxidative stress is also present in patients with ASD (Smaga et al. 2015). High free radical levels cause structural changes in RBC membrane and cytoskeleton, impairing their deformability (Pretorius et al. 2016, Arese et al. 2005, Mohanty et al. 2014).

It was shown that the decrease in deformability and increase in rigidity of RBCs is accompanied by an imbalance of oxidative status, impaired antioxidant enzymes in RBCs and oxidative alteration of RBC membranes in children with ASD (László et al. 2013). Other authors found that autistic children have decreased RBC membrane fluidity, decreased activity of $\mathrm{Na}, \mathrm{K}$-ATPase in RBC membranes and higher levels of lipid peroxidation products in RBCs. In addition, they described a relationship between hyperactivity in ASD and some parameters of the lipidomic profile and membrane fluidity of RBCs (Ghezzo et al. 2013). Chauhan et al. (2004) observed decreased levels of phosphatidylethanolamine and increased phosphatidylserine in RBC membranes of autistic children compared with their non-autistic siblings and suggested that higher oxidative stress in autism leads to externalization of phosphatidylserine. This mechanism leads to eryptosis, e.g. apoptosis-like cell death of RBCs (Qadri et al. 2017). In addition, activities of antioxidant enzymes such as superoxide dismutase, catalase, glutathione reductase, and glutathione peroxidase are decreased in autistic children (Meguid et al. 2011). Interestingly, in vitro incubation of RBCs from subjects with ASD with antioxidants normalized their morphology (Bolotta et al. 2018).

As we have found significant correlation neither between MCV and RBC deformability nor between MCV and scores in $\mathrm{C}$ domain (as well as its subdomains), we can conclude that differences in RBC deformability are not consequences of differences in $\mathrm{MCV}$ in our participants.

In our study, we found significant correlation between RBC deformability and restrictive, repetitive, and stereotyped behaviors and interests, more specifically subdomains focused on preoccupations, circumscribed interests and adherence to routines. However, we were unable to find correlation between RBC deformability and other two subdomains of this domain. This may be in agreement with several author's findings showing that behavioral domain of restricted and repetitive behaviors and interests (domain C) is comprised of more subcategories (Bishop 2013, Tao et al. 2016), with different underlying etiology. It may be important for future research to better conceptualize the clinical subtypes of ASD when searching for its biomarkers, as different biomarkers can contribute to a variety of behavioral symptoms of ASD.

We are aware of potential limitations of our study. Relatively small group of participants was included and we were not able to guarantee their identical life-style including diet. Although, our statistically significant correlation coefficients show only weak negative relations between $\mathrm{RBC}$ deformability and individual scores reflecting ASD symptomatology, we suggest that determination of RBC deformability could have an independent and powerful contribution in multivariate predictive models that assess severity of ASD.

\section{Conclusions}

Abnormalities in RBC deformability may at least partially be involved in pathomechanisms of ASD and contribute to its clinical manifestation. $\mathrm{RBC}$ deformability has a potential to become a clinical tool that could be used as peripheral biomarker of ASD in terms of its earlier diagnosis or prediction of prognosis. Moreover, further research is necessary in order to bring more data and to establish if RBC deformability can serve as prognostic biomarker in ASD.

\section{Conflict of Interest}

There is no conflict of interest.

\section{Acknowledgements}

Supported by grants VEGA 1/0286/18, APVV-15-0085 and APVV-15-0045. The authors thank Dagmar Ciganekova, Helena Bacigalova and Jana Meryova for their excellent technical assistance. 


\section{References}

ABDELMEGUID NE, KOURTIAN SG: Subcellular disorders associated with autism peripheral blood. Asian J Biol Sci 5: 221-239, 2012.

AMERICAN PSYCHIATRIC ASSOCIATION: Diagnostic and Statistical Manual of Mental Disorders: DSM-5. American Psychiatric Association, Washington, D.C, 2013.

ARESE P, TURRINI F, SCHWARZER E: Band 3/complement-mediated recognition and removal of normally senescent and pathological human erythrocytes. Cell Physiol Biochem 16: 133-146, 2005.

BABINSKÁ K, BUCOVÁ M, ĎURMANOVÁ V, LAKATOŠOVÁ S, JÁNOŠÍKOVÁ D, BAKOŠ J, HLAVATÁ A, OSTATNÍKOVÁ D: Increased plasma levels of the high mobility group box 1 protein (HMGB1) are associated with a higher score of gastrointestinal dysfunction in individuals with autism. Physiol Res 63: 613-618, 2014.

BABINSKÁ K, TOMOVA A, CELUŠÁKOVÁ H, BABKOVÁ J, REPISKÁ G, KUBRANSKÁ A, FILČÍKOVÁ D, SIKLENKOVÁ L, OSTATNÍKOVÁ D: Fecal calprotectin levels correlate with main domains of the autism diagnostic interview-revised (ADI-R) in a sample of individuals with autism spectrum disorders from Slovakia. Physiol Res 66: 517-522, 2017.

BASKURT OK, MEISELMAN HJ: Blood rheology and hemodynamics. Semin Thromb Hemost 29: 435-450, 2003.

BISHOP SL, HUS V, DUNCAN A, HUERTA M, GOTHAM K, PICKLES A, KREIGER A, BUJA A, LUND S, LORD C: Subcategories of restricted and repetitive behaviors in children with autism spectrum disorders. Autism Dev Disord 43: 1287-1297, 2013.

BOLOTTA A, BATTISTELLI M, FALCIERI E, GHEZZO A, MANARA MC, MANFREDINI S, MARINI M, POSAR A, VISCONTI P, ABRUZZO PM: Oxidative stress in autistic children alters erythrocyte shape in the absence of quantitative protein alterations and of loss of membrane phospholipid asymmetry. Oxid Med Cell Longev 2018: 6430601, 2018.

CHANG CY, LIANG HJ, CHOW SY, CHEN SM, LIU DZ: Hemorheological mechanisms in Alzheimer's disease. Microcirculation 14: 627-634, 2007.

CHAUHAN V, CHAUHAN A, COHEN IL, BROWN WT, SHEIKH A: Alteration in amino-glycerophospholipids levels in the plasma of children with autism: A potential biochemical diagnostic marker. Life Sci 74: 1635-1643, 2004.

CICCOLI L, DE FELICE C, PACCAGNINI E, LEONCINI S, PECORELLI A, SIGNORINI C, BELMONTE G, GUERRANTI R, CORTELAZZO A, GENTILE M, ZOLLO G, DURAND T, VALACCHI G, ROSSI M, HAYEK J: Erythrocyte shape abnormalities, membrane oxidative damage, and $\beta$-actin alterations: an unrecognized triad in classical autism. Mediators Inflamm 2013: 432616, 2013.

DIEZ-SILVA M, DAO M, HAN J, LIM CT, SURESH S: Shape and biomechanical characteristics of human red blood cells in health and disease. MRS Bull 35: 382-388, 2010.

EDMISTON E, ASHWOOD P, VAN DE WATER J: Autoimmunity, autoantibodies, and autism spectrum disorder. Biol Psychiatry 81: 383-390, 2017.

GHEZZO A, VISCONTI P, ABRUZZO PM, BOLOTTA A, FERRERI C, GOBBI G, MALISARDI G, MANFREDINI S, MARINI M, NANETTI L, PIPITONE E, RAFFAELLI F, RESCA F, VIGNINI A, MAZZANTI L: Oxidative stress and erythrocyte membrane alterations in children with autism: correlation with clinical features. PLoS One 8: e66418, 2013.

KIM J, LEE H, SHIN S: Advances in the measurement of red blood cell deformability: A brief review. $J$ Cell Biotechnol 1: 63-79, 2015.

KUSHAK RI, BUIE TM, MURRAY KF, NEWBURG DS, CHEN C, NESTORIDI E, WINTER HS: Evaluation of intestinal function in children with autism and gastrointestinal symptoms. J Pediatr Gastroenterol Nutr 62: 687-691, 2016.

LÁSZLÓ A, NOVÁK Z, SZÖLlÖSI-VARGA I, HAI DU Q, VETRÓ Á, KOVÁCS A: Blood lipid peroxidation, antioxidant enzyme activities and hemorheological changes in autistic children. Ideggyogy Sz 66: 23-28, 2013.

LIM H, BACK SM, NAM J, CHOI H: Determination of red blood cell deformability using centrifugal force in a threedimensional-printed mini-disk (3D-PMD). PLoS One 13: e0197619, 2018. 
LORD C, RUTTER M, DILAVORE PC, RISI S, GOTHAM K, BISHOP S: Autism Diagnostic Observation Schedule, Second Edition (ADOS-2) Manual (Part I): Modules 1-4. Torrance, CA: Western Psychological Services, 2012.

LORD C, RUTTER M, LE COUTEUR A: Autism diagnostic interview-revised: a revised version of a diagnostic interview for caregivers of individuals with possible pervasive developmental disorders. J Autism Dev Disord 24: 659-685, 1994.

LYALL K, CROEN L, DANIELS J, FALLIN MD, LADD-ACOSTA C, LEE BK, PARK BY, SNYDER NW, SCHENDEL D, VOLK H, WINDHAM GC, NEWSCHAFFER C: The changing epidemiology of autism spectrum disorders. Annu Rev Public Health 38: 81-102, 2017.

MAZUREK M: The history of comorbidity in autism spectrum disorders (ASD). In: Comorbid Conditions Among Children with Autism Spectrum Disorders. MATSON J (ed.) Autism and Child Psychopathology Series, Springer, Cham, 2016, pp 3-25.

MEGUID NA, DARDIR AA, ABDEL-RAOUF ER, HASHISH A: Evaluation of oxidative stress in autism: defective antioxidant enzymes and increased lipid peroxidation. Biol Trace Elem Res 143: 58-65, 2011.

MOHANTY JG, NAGABABU E, RIFKIND JM: Red blood cell oxidative stress impairs oxygen delivery and induces red blood cell aging. Front Physiol 5: 84, 2014.

MUSKENS JB, VELDERS FP, STAAL WG: Medical comorbidities in children and adolescents with autism spectrum disorders and attention deficit hyperactivity disorders: a systematic review. Eur Child Adolesc Psychiatry 26: 1093-1103, 2017.

PARTHASARATHI K, LIPOWSKY HH: Capillary recruitment in response to tissue hypoxia and its dependence on red blood cell deformability. Am J Physiol 277: H2145-H2157, 1999.

PRETORIUS E, OLUMUYIWA-AKEREDOLU OO, MBOTWE S, BESTER J: Erythrocytes and their role as health indicator: Using structure in a patient-orientated precision medicine approach. Blood Rev 30: 263-274, 2016.

QADRI SM, BISSINGER R, SOLH Z, OLDENBORG PA: Eryptosis in health and disease: A paradigm shift towards understanding the (patho)physiological implications of programmed cell death of erythrocytes. Blood Rev 31: 349-361, 2017.

RADOSINSKA J, MEZESOVA L, OKRUHLICOVA L, FRIMMEL K, BREIEROVA E, BARTEKOVA M, VRBJAR $\mathrm{N}$ : Effect of yeast biomass with high content of carotenoids on erythrocyte deformability, NO production and Na,K-ATPase activity in healthy and LPS treated rats. Clin Hemorheol Microcirc 64: 125-134, 2016.

RADOSINSKA J, VRBJAR N: The role of red blood cell deformability and Na,K-ATPase function in selected risk factors of cardiovascular diseases in humans: focus on hypertension, diabetes mellitus and hypercholesterolemia Physiol Res 65: 43-54, 2016.

RICHDALE AL, SCHRECK KA: Sleep problems in autism spectrum disorders: prevalence, nature, \& possible biopsychosocial aetiologies Sleep Med Rev 13: 403-411, 2009.

SCHMID-SCHONBEIN H: Microrheology of erythrocytes, blood viscosity, and the distribution of blood flow in the microcirculation. Int Rev Physiol 9: 1-62, 1976.

SMAGA I, NIEDZIELSKA E, GAWLIK M, MONICZEWSKI A, KRZEK J, PRZEGALIŃSKI E, PERA J, FILIP M: Oxidative stress as an etiological factor and a potential treatment target of psychiatric disorders. Part 2. Depression, anxiety, schizophrenia and autism. Pharmacol Rep 67: 569-580, 2015.

TANASHYAN MM, SHABALINA AA, LAGODA OV, RASKURAZHEV AA, KONOVALOV RN: Multimodal approach to treatment of neurological complications of chronic brain ischemia. Ter Arkh 90: 61-67, 2018.

TAO Y, GAO H, ACKERMAN B, GUO W, SAFFEN D, SHUGART YY: Evidence for contribution of common genetic variants within chromosome $8 \mathrm{p} 21.2-8 \mathrm{p} 21.1$ to restricted and repetitive behaviors in autism spectrum disorders. BMC Genomics 17: 163, 2016. 